\title{
PLANEJAMENTO DE REPARO BASEADO EM INSPEÇÃO DE SEGURANÇA NR-13 E SISTEMA DE GERENCIAMENTO DE INTEGRIDADE ESTRUTURAL*
}

\author{
Kallin de Souza ${ }^{1}$ \\ José Gonçalves Pacheco Junior ${ }^{2}$ \\ Igor Martins dos Santos ${ }^{3}$
}

\section{Resumo}

No âmbito da inspeção de segurança de caldeiras, a norma regulamentadora NR-13 estabelece a necessidade de um sistema de gerenciamento de integridade estrutural (SGI) com requisitos mínimos para atestar a segurança do equipamento. Entretanto, visando estabelecer um SGI que possibilite maior acompanhamento do equipamento e melhor direcionamento dos reparos instaurou-se um sistema de inspeção com procedimentos mais abrangentes. Esse trabalho apresenta as técnicas de ensaio não destrutivo (END) utilizadas usualmente na inspeção de segurança de NR-13 e novas como o ensaio IRIS (Internal Rotary Inspection System) [1]. As condições estruturais foram comparadas com as recomendadas segundo os códigos de projetos internacionais. Além disso, técnicas de estatística de engenharia de manutenção e projeto foram utilizadas, permitindo quantificar a segurança de operação e programar o momento da intervenção para o reparo.

Palavras-chave: Caldeira; NR-13; Sistema de gestão de integridade estrutural; Inspeção

\section{REPAIR PLANNING BASED ON SAFETY INSPECTION NR-13 AND STRUCTURAL INTEGRITY MANAGEMENT}

\section{Abstract}

On steam boilers safety inspection, the regulatory standard NR-13 establishes a structural integrity management with minimum requirements to guarantee steam boilers reliability. However, aiming a complete Structural Integrity Management (SIM), which could provide the highest quality information that is useful to conduct a repair, a new inspection approach was used. This paper presents non-destructives testing (NDT) that had been used in the safety inspection and new ones like IRIS (Internal Rotary Inspection System) [1]. International standards were used to evaluate the actual condition found during the integrity evaluation. Probability concepts in engineering were also used to assure operational condition of the boiler and planning an up come repair.

Keywords: Steam boiler; Brazilian standard NR-13; Structural integrity management.

1 Engenheira mecânica, Engenheira de manutenção Mecânica I, Engenharia de manutenção \& expertise/manutenção central, thyssenkrupp CSA Siderúrgica do Atlântico, Rio de Janeiro, Rio de Janeiro e Brasil.

2 Engenheiro Mecânico, Especialista, Profissional Habilitado NR13, Engenharia de Manutenção \& Expertise/Manutenção Central, thyssenkrupp CSA Siderúrgica do Atlântico, Rio de Janeiro, Rio de Janeiro e Brasil.

3 Engenheiro Mecânico, Engenheiro de Manutenção Mecânica, Gerência de Distribuição de Energia e Utilidades, thyssenkrupp CSA Siderúrgica do Atlântico, Rio de Janeiro, Rio de Janeiro e Brasil. 


\section{INTRODUÇÃO}

Caldeiras são equipamentos que podem acarretar acidentes de grandes proporções em função do risco de explosões e rupturas de partes, uma vez que esses equipamentos trabalham sob elevadas pressões e temperaturas.

$\mathrm{Na}$ última década cerca de dez acidentes por ano envolvendo caldeiras são reportados na mídia. O número de acidentes fatais envolvendo tal equipamento atingiu seu ápice em 1865 com o acidente na caldeira da embarcação Sultana, cerca de 1800 vítimas fatais foram contabilizadas. Em função dessa e de inúmeras ocorrências que se seguiram a ASME (American Society of Mechanical Engineers) formalizou no começo do século XX o código de construção de caldeiras e vasos de pressão, levando a uma sensível redução de acidentes [2].

Contudo, no presente, a principal causa de acidentes e ou incidentes tem se revelado a manutenção e operação inadequada do ativo. Nesse âmbito foi necessária a presença de normas de segurança que regulamentem esses equipamentos.

No Brasil tem-se a NR-13, norma regulamentadora, que estabelece requisitos mínimos para gestão de integridade estrutural de vasos de pressão, caldeiras a vapor e tubulações, englobando os aspectos de instalação, operação e funcionamento $[3,4]$.

De modo a garantir, com maior segurança, os requisitos levantados pelos órgãos regulamentadores e aperfeiçoar as intervenções de manutenção, assegurando com que os riscos estejam abaixo dos limites aceitáveis e por consequência garantir disponibilidade do equipamento, pode-se ir além do que a norma prevê, estabelecendo um sistema de gestão de integridade estrutural mais robusto avaliando a probabilidade de falha e consequência de falha.

O sistema de gestão de integridade estrutural (SGI) abrange cinco competências. São elas, gestão, inspeção e integridade, manutenção, operação e processo. Sendo Inspeção a base da estrutura organizacional, uma vez que fornece dados para fundamentar as decisões dos demais setores.

A inspeção e integridade no universo do SGI abrange a definição de ameaças e mecanismos de danos que possam existir e os procedimentos e técnicas necessários para a avaliação e monitoramento dos mesmos, levando em consideração a qualidade e frequência dos dados obtidos de modo a alimentar a matriz de análise de risco que norteia os procedimentos de manutenção.

Segundo French [5,6], corrosão por fadiga térmica e corrosão estão entre os principais mecanismos de danos em caldeiras. Várias técnicas podem ser utilizadas para quantificar o dano gerado e monitorar o mesmo. Entre elas podemos citar os métodos de ensaios não destrutivos, de medição de espessura por ultrassom convencional e medição de espessura por IRIS (Internal Rotary Inspection System).

O princípio de funcionamento da medição de espessura por ultrassom constitui no método de pulso ultrassônico e eco. No medidor convencional as medições são feitas de forma pontual sendo necessário, portanto, determinar os pontos críticos onde se espera maior perda de espessura.

Apesar de conferir um indicador para perda de espessura esse método não confere uma visão global sobre a tubulação. A presença de pitis um pouco mais profundos na parede de um tubo já pode ser o suficiente para que o mesmo seja trocado, logo é necessário um método mais abrangente. O método de ensaio IRIS, utilizado com sucesso em indústrias de óleo e gás, realiza a medição de espessura em toda a 
seção do tubo e ao longo do seu comprimento, de modo a produzir uma imagem planificada do mesmo.

Nesse contexto o presente trabalho tem como objetivo descrever a abordagem de ensaios utilizada durante inspeção de segurança de NR-13 de uma caldeira aquatubular, de categoria $A$, pertencente à planta de uma usina siderúrgica, que visam estabelecer dados mais confiáveis e abrangentes em relação ao equipamento, permitindo melhorar o sistema de gerenciamento de integridade existente e, por consequência, assegurar maior confiabilidade ao garantir a integridade, o procedimento de reparo e a segurança do ativo. Fatores esses que garantem maior disponibilidade do equipamento $e$, consequentemente, maior estabilidade dos processos vinculados a este.

\section{MATERIAIS E MÉTODOS}

A aplicação dos seguintes tipos de ensaios não destrutivos foi utilizada durante a avaliação de integridade e segurança da caldeira.

\subsection{Inspeção Visual}

Tanto na região interna quanto externa da caldeira a inspeção visual conduzida teve por objetivo identificar danos causados por corrosão, indicativos de deformação e vazamentos.

\subsection{Medição de espessura por ultrassom convencional}

A medição por ultrassom convencional foi realizada ao longo das paredes da fornalha (Figura 1), direita, esquerda e do fundo. Em todas as paredes os pontos de medição adotados estão localizados a uma cota de 1,2 metros com base no piso da fornalha, cota determinada em função da região de influência da chama do queimador. Toda a extensão das paredes foi medida. Além desses pontos nas paredes direita e esquerda foram ainda medidos os pontos na curvatura da tubulação (Figura 2), nesse caso 34 tubos, partindo da região mais próxima do queimador, foram mapeados de cada lado.

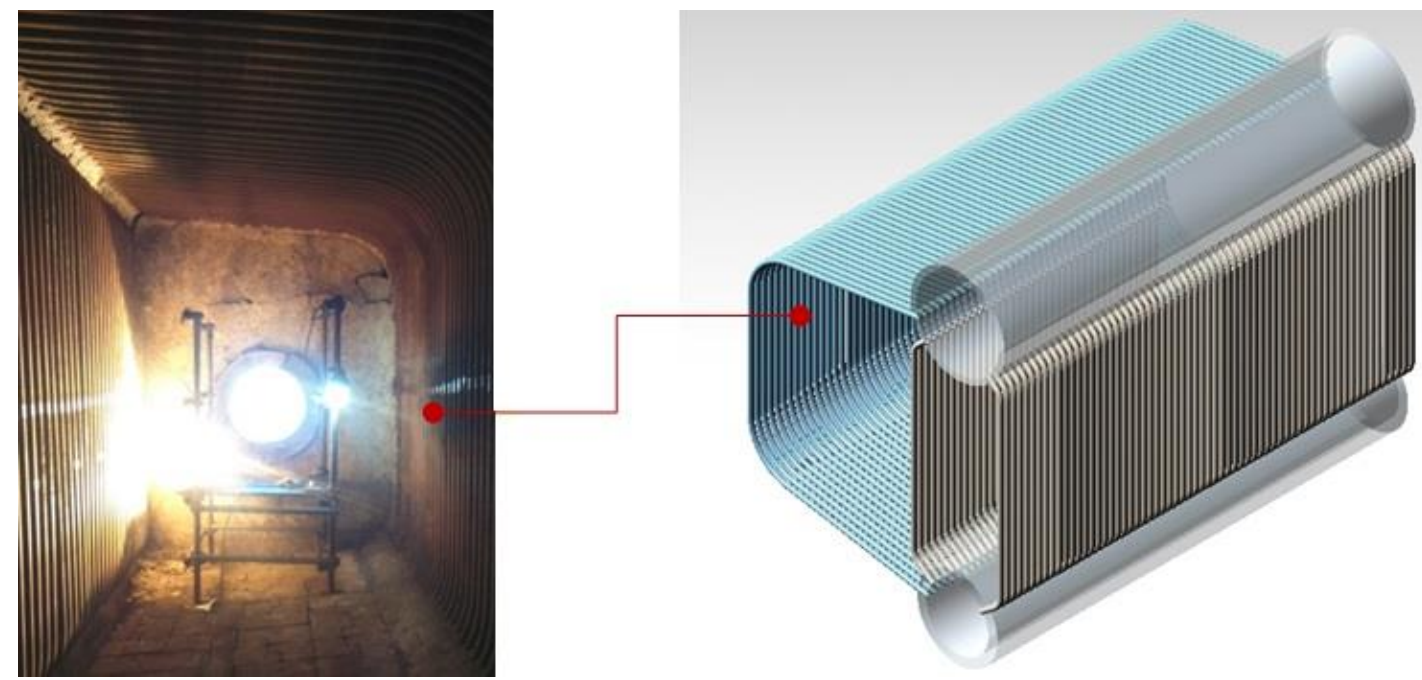

Figura 1. Paredes da fornalha. 


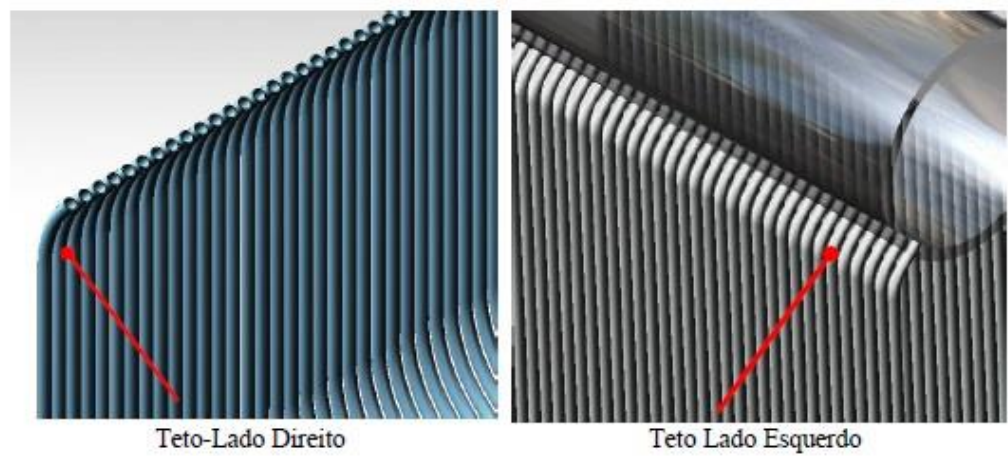

Figura 2. Pontos de Medição Próximos ao teto da fornalha.

\subsection{Medição de espessura por IRIS.}

A metodologia de ensaio por IRIS, que utiliza a técnica de eco por imersão, consiste na emissão de um pulso ultrassônico por meio de uma sonda alocada no centro da tubulação de interesse, este preenchido por água. $O$ pulso se propaga no meio aquoso, uma parcela é refletida pela parede externa e a outra, que propagou através da parede do tubo é refletida pela extremidade exterior. A sonda capta os dois pulsos oriundos da reflexão e contabiliza a diferença entre os tempos de captação do mesmo, desse modo determina a espessura da parede. Conforme a sonda rotaciona e translada, a espessura ao longo da secção tubular e do comprimento é registrada $[1,7]$.

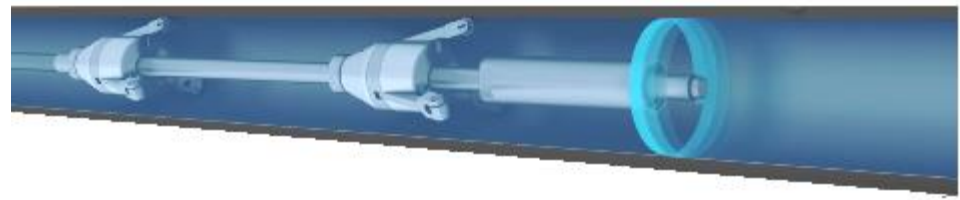

Figura 3. Esquema da Sonda Iris [1].

O ensaio de IRIS foi realizado em 35 tubos da parede de água, ao longo de 3 metros de comprimento da tubulação. Entre eles foram registrados três gamas de espessuras diferentes (Figura 4)

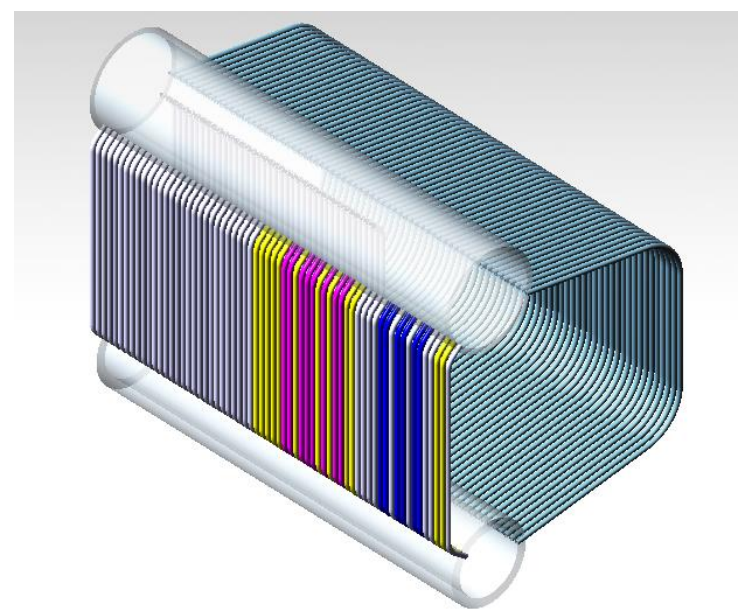

Figura 4. Representação das tubulações com medição realizada pelo ensaio IRIS. 


\subsection{Réplica Metalográfica e Dureza.}

Visando avaliar os efeitos causados pela exposição a altas temperaturas do material foi realizado o ensaio de réplica metalográfia e dureza em quatro pontos, 3 pontos na tubulação que compõe o superaquecedor e 1 ponto na fornalha, onde havia sido identificado forte marca de chama na parede.

Esse ensaio consiste em preparar a superfície de interesse com uma sequência de polimentos seguidos de ataque químico para revelar a microestrutura do material. A microestrutura revelada é "copiada" em uma lamina de acetato, a qual é examinada posteriormente no laboratório.

Já a dureza é aferida, na mesma região onde a réplica foi realizada, mediante a utilização de um durometro portátil.

\section{RESULTADOS E DISCUSSÃO}

O exame visual levou a constatação de considerável deformação na tubulação referente ao superaquecedor, processo de corrosão nos tubulões de água e vapor, esse último com maior intensidade, além de marcas de chama nas paredes próximas ao queimador.

A condição apresentada indica a necessidade da troca da tubulação do superaquecedor, entretanto a mesma pode ser conduzida em dois anos, no próximo ciclo de inspeção. A análise metalográfica e de dureza indicaram as condições do material como aceitáveis e a deformação apesar de severa não compromete a segurança dentro do prazo de operação considerado.
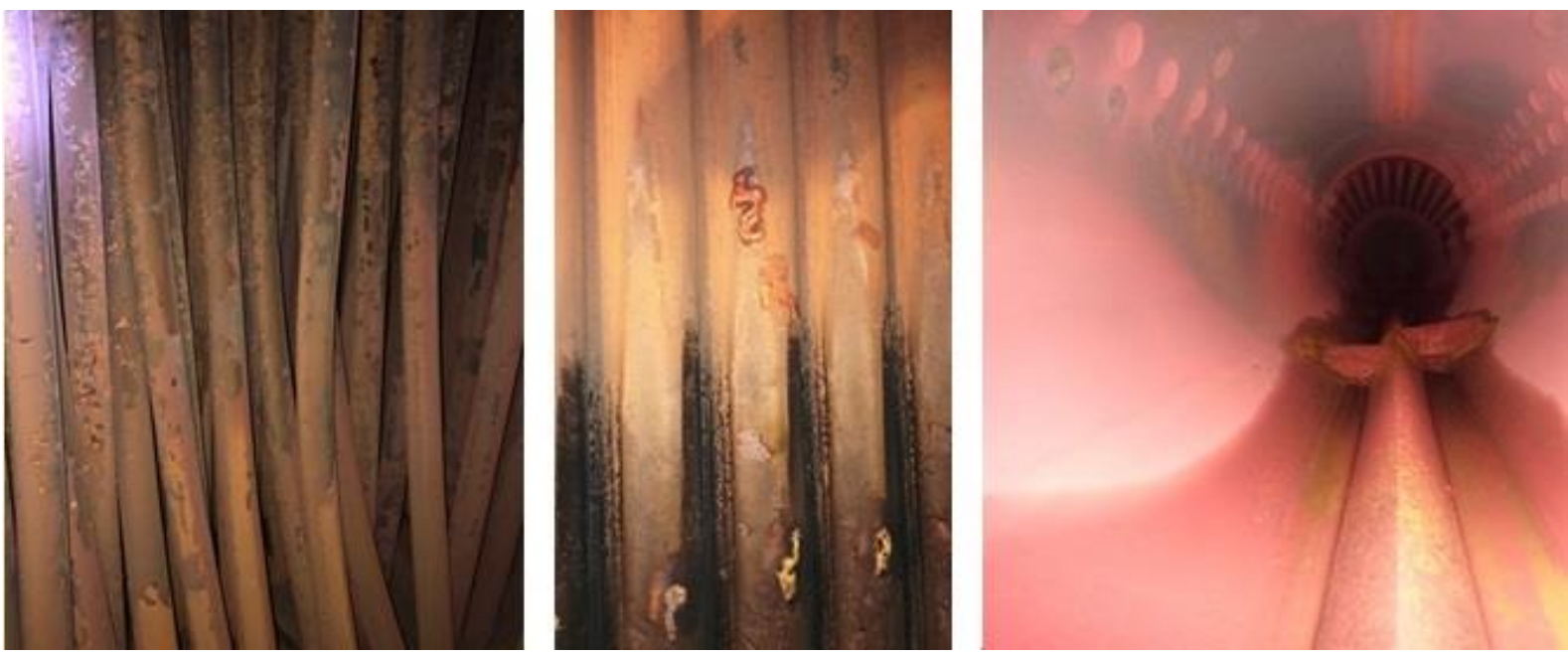

Figura 5. Inspeção Visual- Superaquecedor à esquerda, parede direita interior fornalha figura central e tubulão de vapor à direita.

\subsection{Perda de espessura.}

Os resultados da perda de espessura nas paredes da fornalha acompanharam a tendência esperada, maior perda na região onde há maior exposição a altas temperaturas, ou seja, com maior influência das chamas do queimador. Sendo as regiões com maior dispersão de resultados a parede localizada ao fundo (Figura 2) e nas curvas próximas ao teto (Figura 3). 
$\mathrm{Na}$ parede localizada ao fundo da fornalha a maior perda se localiza na região central, consistente com a região de influência da chama do queimador.

No teto, especificamente nessa região onde se identifica a mudança de direção da tubulação, região curva, houve maior perda de espessura, como esperado, haja vista a maior turbulência do escoamento.

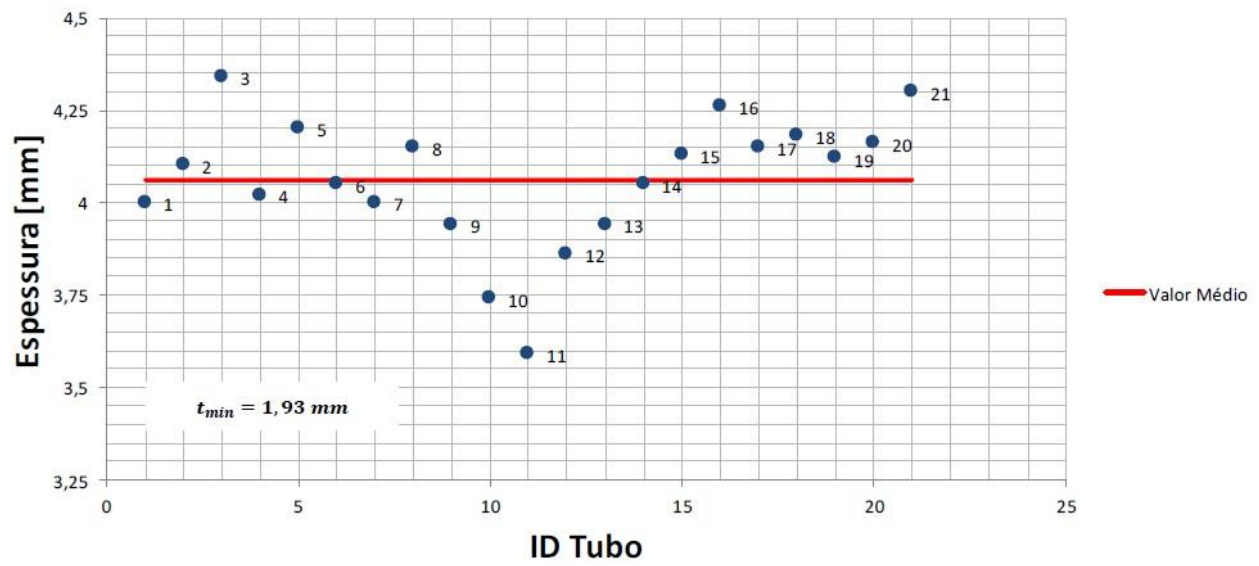

Figura 6. Mapeamento da espessura da fornalha - Parede Traseira.

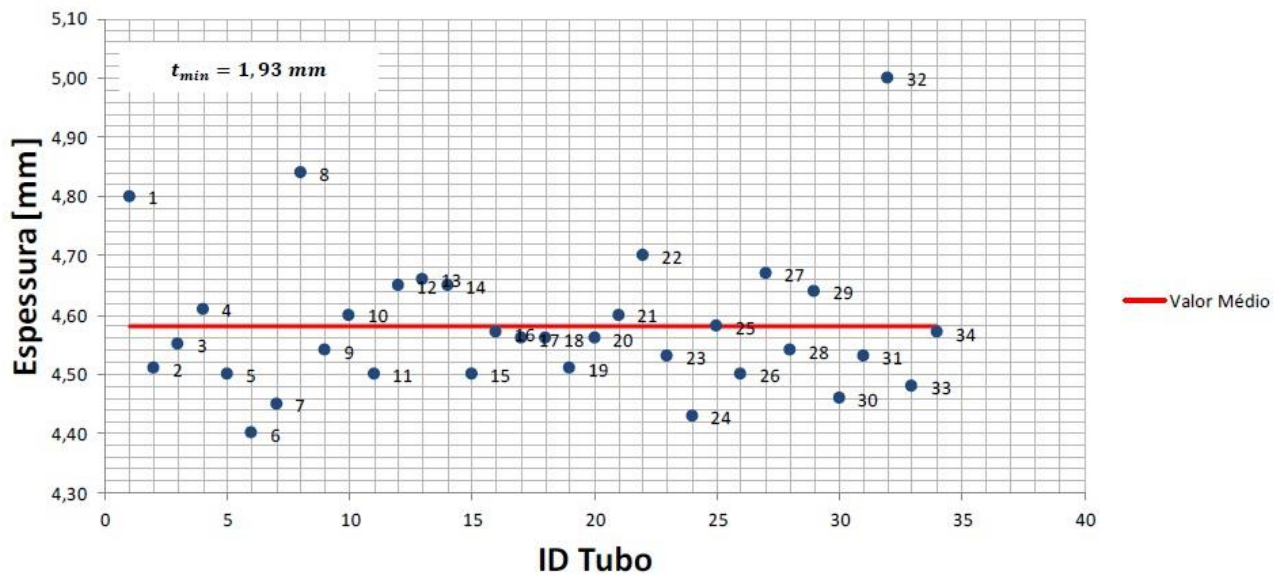

Figura 7. Mapeamento da espessura da fornalha- Curva "Teto" lado esquerdo.

O quadro global da condição da fornalha pode ser exemplificado na figura (Figura 8), por meio da qual podemos analisar a perda de espessura ao longo dos ciclos de inspeção de NR-13. Comparando essa perda com as espessuras mínimas de cada região calculada, a integridade da estrutura pode ser assegurada, com base no tipo de método de medição utilizado. 


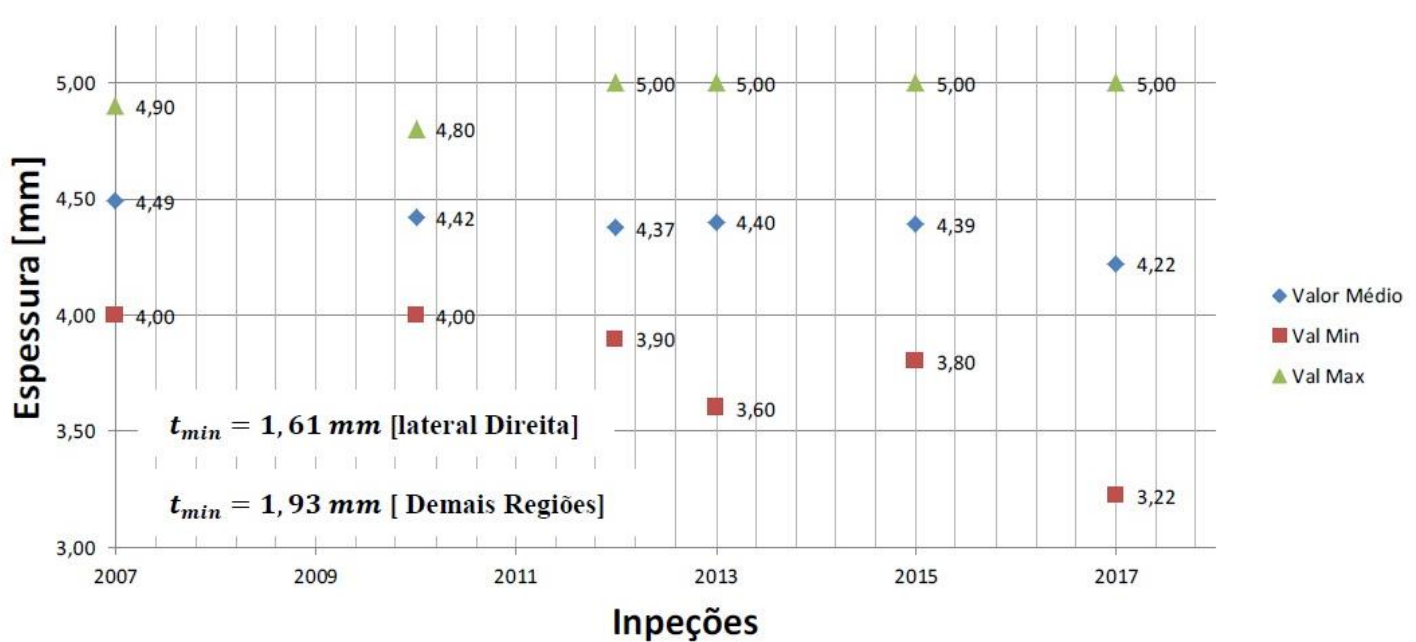

Figura 8. Progressão das avaliações de espessura da fornalha ao longo dos anos.

\subsubsection{Cálculo de Confiabilidade em trecho com severa perda de espessura.}

Devido à impossibilidade de acessar o feixe tubular de água e vapor (riser), conectados aos tubulões, o método IRIS foi 0 indicado para a inspeção nesse trecho. Revelando um cenário alarmante. A perda de espessura identificada nos tubos examinados foi considerada severa, os tubos assinalados com a cor rosa no mapa (Figura 6) apresentam espessura na faixa de 2,6 a $1,8 \mathrm{~mm}$, a espessura mínima é $1,61 \mathrm{~mm}$.

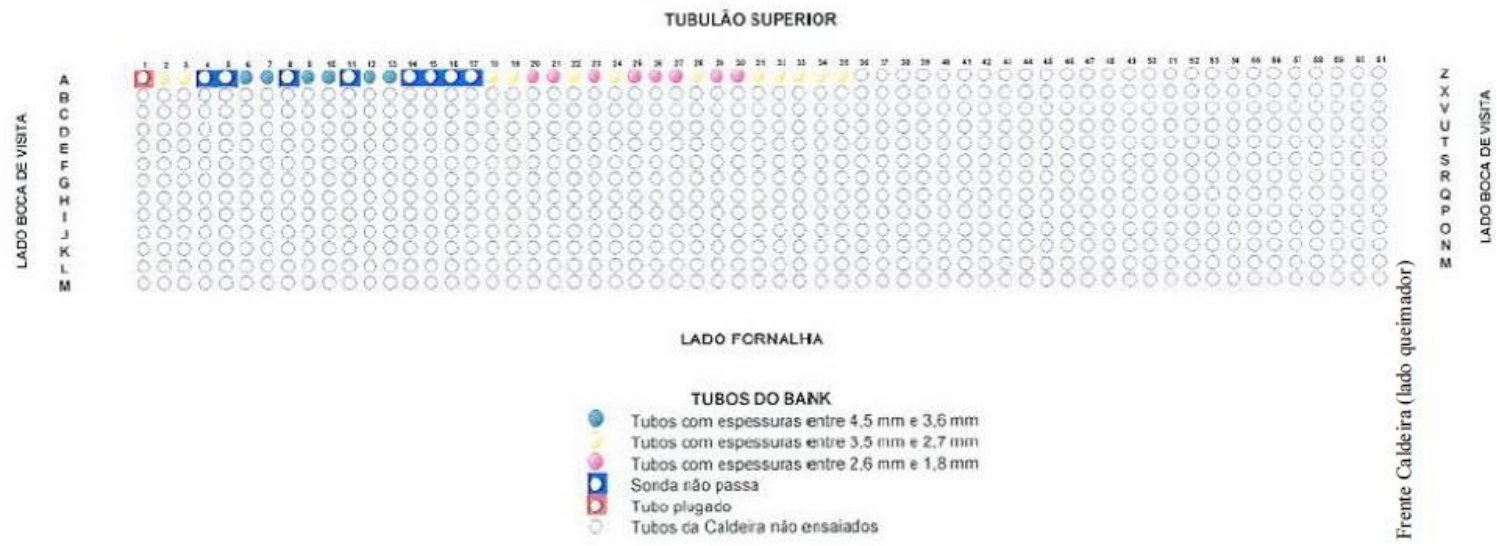

Figura 9. Mapeamento do Ensaio IRIS-Relatório Cieme Engenharia Eireli.

Para estabelecer um critério de aceitação e, portanto, a análise de risco visando à recomendação para execução de reparo, foi considerado o conceito de confiabilidade estrutural ou probabilidade de falha. $\mathrm{O}$ aspecto básico é garantir que a resistência (Capacidade) seja superior ao carregamento (Demanda). Para estabelecer tal relação utiliza-se a variável margem (Equação 1):

$$
m=C-D
$$

Admitindo-se que as variáveis "C" e "D" pertençam a distribuições estatísticas com médias e desvio padrões, a variável margem também o será (Equação 2). Ocorrerá, por conseguinte, uma falha quando a margem for inferior a zero e a probabilidade de falha consiste na área sobre a curva de distribuição utilizada $[8,9,10]$. 


$$
s_{m}=\sqrt{s_{C}^{2}+s_{D}^{2}}
$$

A metodologia nesse trabalho considera como variáveis de Capacidade e Demanda, a tensão admissível $(S)$ e a tensão de trabalho $(\sigma)$ (Equação 3) respectivamente. Sendo a perda de espessura por corrosão ao longo do tempo (Equação 4) o mecanismo de dano definido como principal.

$$
\begin{array}{r}
\sigma=\frac{P}{w}\left[\frac{D-(t-0.005 D-e)}{2 t-0.01 D-2 e}\right] \\
t=t_{o}-\operatorname{ar}(4)
\end{array}
$$

Os dados levantados mostraram que a população é gaussiana. Dessa maneira, a análise estatística empregada é baseada na distribuição gaussiana, ou normal. Na tabela (Tabela 1) estão descritos as variáveis empregadas bem como as incertezas calculadas.

Tabela 1. Variáveis - Parede de água

\begin{tabular}{lcc}
\hline Descrição de Variável & Valor & Desvio Padrão \\
\hline P- Pressão PMTA [MPa] & 2.75 & 0.8 \\
\hline D- Diâmetro Externo [mm] & 63.5 & 0 \\
\hline $\boldsymbol{t}_{\boldsymbol{o}}$ - Espessura mínima medida [mm] & 2.1 & 0.72 \\
\hline a- anos & $2-4$ & 0 \\
\hline r- taxa de corrosão [mm/ano] & 0.085 & 0 \\
\hline w-Fator de Junta & 1 & 0 \\
\hline $\boldsymbol{S}$-Tensão admissível & 67.522 & 0.01 \\
\hline e- fator de espessura & 0 & 0 \\
\hline $\boldsymbol{s}_{\boldsymbol{\sigma}}(\boldsymbol{a}=\mathbf{4})$ - Incerteza tensão adm & 34.73 & - \\
\hline $\boldsymbol{s}_{\boldsymbol{\sigma}}(\boldsymbol{a}=\mathbf{2})$ - Incerteza tensão adm & 28.61 & - \\
\hline
\end{tabular}

Considerando dois anos de operação, que constitui um ciclo de inspeção de segurança, segundo a NR-13 item 13.4.4.4 aliena c, para a primeira simulação a probabilidade de falha, $\operatorname{Pf}(m<0)$, determinada foi de $30,21 \%$, como consequência a confiabilidade, $R$, para a manutenção da tubulação seria de $69,78 \%$. 


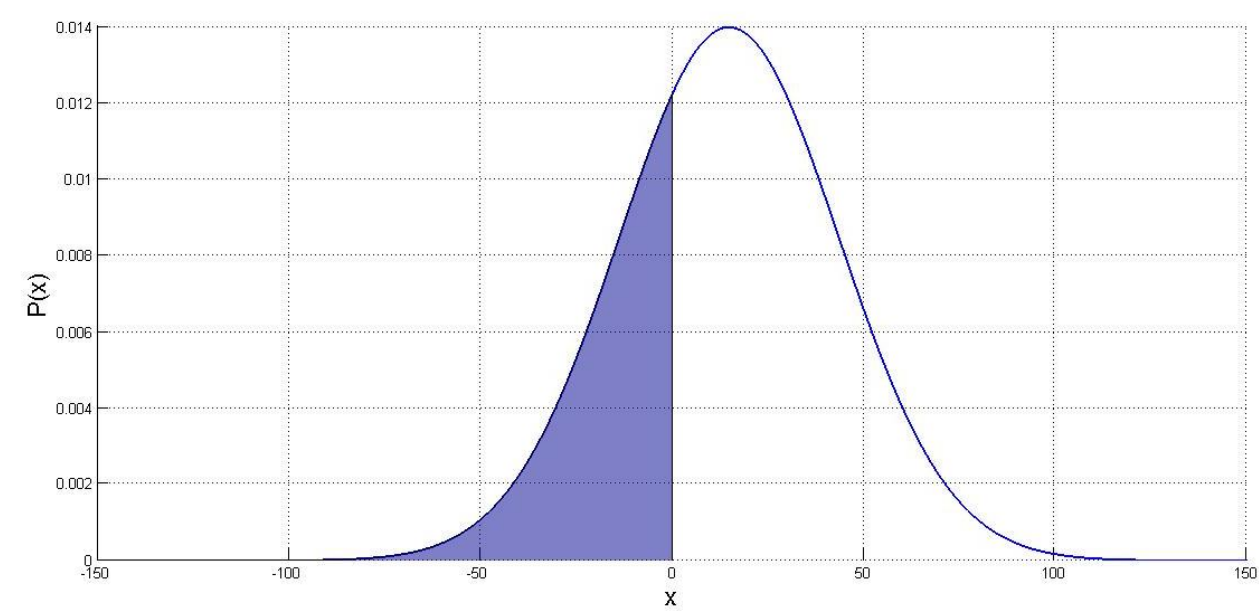

Figura 10. Distribuição normal com área equivalente a probabilidade de falha em destaque Probabilidade de Falha em 2 anos.

Entretanto, quando foi realizado o procedimento com mais um ciclo de inspeção NR13, 4 anos de operação. O cenário constata a impossibilidade de seguir a operação sem a realização da troca do riser. Observa-se nesse caso uma sensível redução no índice de confiabilidade, $59,61 \%$. O que indica que o reparo deve ser programado ao longo do intervalo dos dois anos para a próxima inspeção de NR-13.

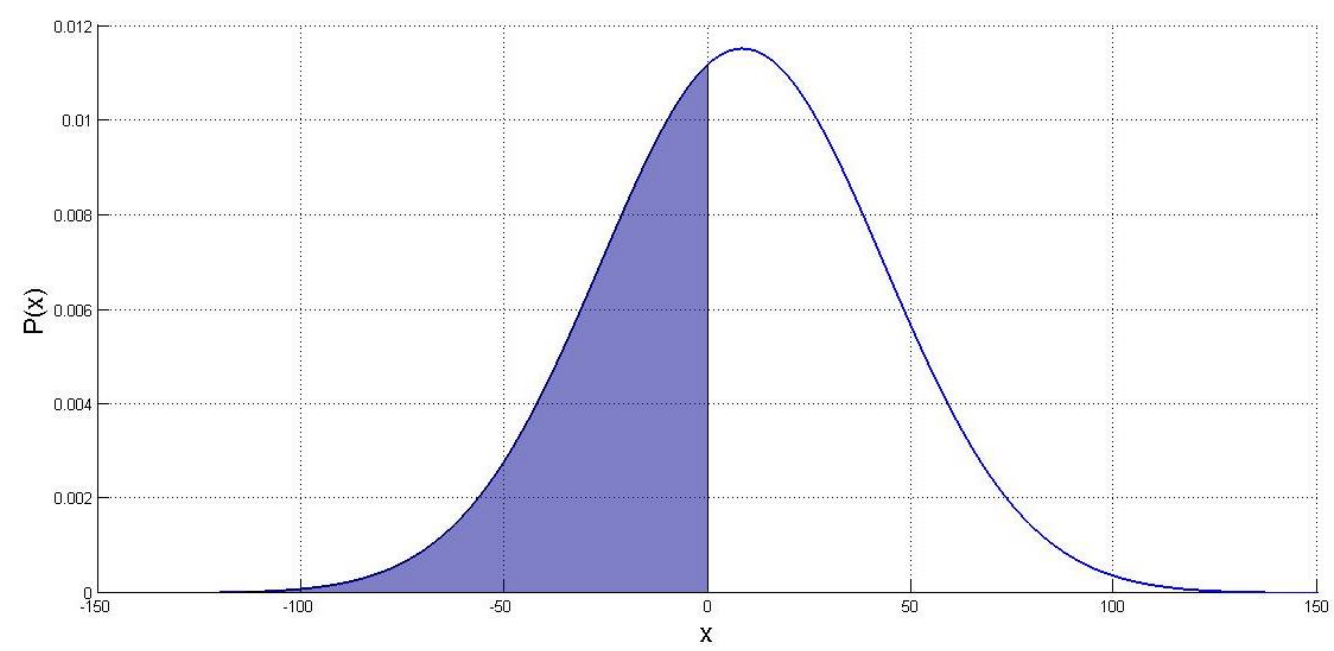

Figura 11. Distribuição normal com área equivalente a probabilidade de falha em destaque Probabilidade de Falha em 4 anos.

\section{CONCLUSÃO}

Esse trabalho conduziu uma avaliação da integridade estrutural de uma caldeira aquatubular por meio de técnicas de inspeção que possibilitaram determinar a confiabilidade do equipamento para o período de dois anos. Foram estabelecidos ainda pontos que devem sofrer reparo ao final da validade da inspeção de segurança. 
Deverá ser programada a troca dos tubos da parede de água com espessura entre $2,6 \mathrm{~mm}$ e $1,8 \mathrm{~mm}$ e conduzida uma reavaliação nas condições dos demais. Assim como a seção tubular do superaquecedor deverá ser refeita.

As técnicas de análise utilizadas permitiram criar e alimentar um sistema de gerenciamento de integridade estrutural mais abrangente para o ativo.

Um ponto fundamental desse trabalho foi a utilização da técnica de inspeção IRIS. Esse tipo de ensaio possibilitou verificar a condição de uma seção do equipamento não inspecionada anteriormente pela dificuldade de acesso. Possibilitando identificar uma região crítica no equipamento, e a probabilidade de ocorrência de uma falha, furo na tubulação, proveniente da perda de espessura nessas tubulações.

A utilização de técnicas probabilísticas, cada vez mais comuns em análises estruturais, permite mensurar o risco do equipamento e verificar se o mesmo é aceitável na quantificação da segurança do mesmo. Portanto, colabora na programação do melhor cenário para a realização do reparo.

É possível, portanto, concluir que os procedimentos utilizados para a inspeção conduziram a avaliação da estrutura com níveis de confiança satisfatórios. Todavia, o campo amostral utilizado no ensaio IRIS precisa ser aumentado nas próximas inspeções, visando elevar o nível de precisão das análises provenientes dos dados fornecidos por esse.

A metodologia de inspeção do SGI da caldeira, também possibilitará a programação das intervenções de manutenção de grande porte que deverão ser conduzidas, assegurando um procedimento estruturado para esse evento.

\section{REFERÊNCIAS}

1 MatEval Ltd., (1982) "IRIS - Internal rotary inspection system", Anti-Corrosion Methods and Materials, Vol. 29 Issue: 5,pp.12-14.

2 Heselton KE. Boiler operator's handbook. New York: Fairmont Press; 2004.

3 NR-13 caldeiras, vasos de pressão e tubulações. Portaria MTE n. ${ }^{\circ} 594$, de 28 de abril de 2014.

4 Pereira AD. Tratado de segurança e saúde ocupacional-Aspectos técnicos e jurídicos Volume II NR-13 a NR-15. 2 edição, São Paulo: Saraiva; 2015.

5 French DN. Metallurgical failures in fossil fired boilers. New York: A Wiley-Interscience Publication, John Wiley and Sons Inc.; 2000.

6 Ahmad J, Purbolaksono J, Beng LC.Thermal fatigue and corrosion fatigue in heat recovery area wall side tubes.Engineering Failure Analysis 17 (2010) 334-343.

7 Eddyfi. Institucional sobre inspeção de tubulação.[ acesso em 18 de maio 2017]. Disponível em: http://www.eddyfi.com/technologies/internal-rotary-inspection-systemiris/.

8 Paliga CM, Campos A, Real MV, Diniz SMC. Métodos de análise de confiabilidade aplicados a vigas de concreto armado recuperadas com PRFC.Teoria e Prática na Engenharia Civil, n.17, p.57-66, Maio, 2011.

9 Castro JTP, Meggiolaro MA. Fadiga técnicas e praticas de dimensionamento estrutural sob cargas reais de serviços. Volume II propagação de trincas, efeitos térmicos e estocasticos. Lexington: Amazon; 2011.

10 Évaluation des données de mesure-Guide pour l'expression de l'incertitude de mesure ; 2008. 